\title{
DETERMINAÇÃO DAS PROPRIEDADES HIDRÁULICAS DO SOLO UTILIZANDO TENSIÔMETROS DE POLÍMEROS EM EXPERIMENTOS DE EVAPORAÇÃO ${ }^{(1)}$
}

\author{
Angelica Durigon ${ }^{(2)}$ \& Quirijn de Jong van Lier ${ }^{(3)}$
}

\begin{abstract}
RESUMO
O tensiômetro de polímeros é um equipamento recentemente desenvolvido que permite medir o potencial matricial da água no solo desde a saturação até condições mais secas do que o ponto de murcha permanente. Nesta nota descrevese a utilização de tensiômetros de polímeros em experimentos de evaporação de cilindros com solo deformado, para determinar as propriedades hidráulicas do material. Nos experimentos foi utilizada terra da camada superficial de um Nitossolo Vermelho. O uso de tensiômetros de polímeros em experimentos de evaporação com colunas de solo permitiu a determinação das relações entre condutividade hidráulica, teor de água e potencial matricial na faixa de potenciais matriciais entre -1 e $-150 \mathrm{~m}$.
\end{abstract}

Termos de indexação: tensiômetro de polímeros, condutividade hidráulica, curva de retenção de água.

\section{SUMMARY: DETERMINATION OF SOIL HYDRAULIC PROPERTIES USING} POLYMER TENSIOMETERS IN EVAPORATION EXPERIMENTS

\begin{abstract}
The polymer tensiometer is a newly developed instrument to measure soil water pressure heads from saturation conditions to drier than the permanent wilting point. In this note we describe how the hydraulic properties of disturbed soil samples were determined in laboratory evaporation experiments with these polymer tensiometers. Soil material from the surface layer of an Ultisol was used. The relation among hydraulic conductivity, water content and pressure heads in the range from -1 to $-150 \mathrm{~m}$ could be determined using polymer tensiometers in evaporation experiments.
\end{abstract}

Index terms: polymer tensiometer, hydraulic conductivity, water retention curve.

\footnotetext{
(1) Recebido para publicação em 16 de abril de 2010 e aprovado em 6 de maio de 2011.

(2) Doutoranda do PPG em Física do Ambiente Agrícola, Escola Superior de Agricultura "Luiz de Queiroz" - ESALQ/USP. Caixa Postal 9, Av. Pádua Dias 11, CEP 13418-900 Piracicaba (SP). E-mail: adurigon@esalq.usp.br

(3) Professor do Departamento de Engenharia de Biossistemas, ESALQ/USP. E-mail: qdjvlier@usp.br
} 


\section{INTRODUÇÃO}

O conhecimento das propriedades hídricas (retenção e condução da água) no solo é importante para a modelagem na hidrologia, na meteorologia e na produção vegetal. Sem esse conhecimento, previsões dos fluxos de água no solo e da transpiração vegetal ficam prejudicadas (Šimůnek et al., 1998; Schneider et al., 2006).

Diversos métodos estão disponíveis para determinar as propriedades hídricas do solo (Dane \& Topp, 2002). Um deles é o "método de evaporação", aplicado a colunas de solo (Wind, 1968; Schindler, 1980; Wendroth et al., 1993; Arya, 2002). A distribuição não linear dos perfis de conteúdo de água e potencial matricial durante os experimentos de evaporação define o número ótimo de tensiômetros a ser utilizado, bem como as profundidades de instalação (Peters \& Durner, 2008). O método evoluiu quando Schindler (1985) propôs modificações no método original de Wind (1968), medindo a massa total da amostra durante todo o experimento e o potencial matricial em somente duas profundidades. Com isso, foi possível determinar as funções de retenção de água e de condutividade hidráulica simultaneamente.

Para as condições quase-saturadas, por utilizar amostras deformadas, o método não constitui uma alternativa viável, quando comparado com aqueles em que a estrutura é conservada. Para o solo mais seco, no entanto, o método é particularmente interessante pela sua relativa simplicidade. Comparando-o com os experimentos de drenagem ("perfil instantâneo"), o método é muito menos trabalhoso e permite observações no solo mais seco, onde essas observações no solo sob drenagem livre são geralmente inviáveis (De Jong van Lier \& Libardi, 1999).

Uma das limitações desse método advém do uso de tensiômetros convencionais, cuja funcionalidade está geralmente limitada a potenciais matriciais entre 0 e -9 m. Essa limitação está ligada não somente ao método específico, como também a qualquer método experimental que emprega tensiômetros. No método em discussão, pelo fato de se adequar a observações no solo mais seco, essa limitação é especialmente prejudicial. Um novo tipo de tensiômetro - o tensiômetro de polímeros - foi recentemente desenvolvido (Bakker et al., 2007; van der Ploeg et al., 2008; De Rooij et al., 2009). Este tensiômetro é capaz de medir a tensão da água retida no solo em toda a faixa agronômica e ecologicamente interessante, desde a saturação $(h=0 \mathrm{~m})$ até $h=-200 \mathrm{~m}$, além do ponto de murcha permanente, considerado normalmente em $h=-150 \mathrm{~m}$. A utilização de tensiômetros de polímeros num esquema experimental análogo ao proposto por Schindler (1985) permitiria, portanto, as medidas simultâneas das propriedades hidráulicas do solo na faixa completa de conteúdos de água de interesse em estudos ambientais e agronômicos.
Nesta nota é descrita a adaptação do método de Schindler et al. (1985) para uso com tensiômetros de polímeros e discute-se a aplicação do método, com base num teste realizado para caracterizar hidraulicamente uma amostra deformada de terra de um Nitossolo Vermelho.

\section{ASPECTOS TEÓRICOS}

\section{Condutividade hidráulica}

Considera-se uma coluna vertical preenchida com material de solo de altura $L(\mathrm{~m})$ e área $A\left(\mathrm{~m}^{2}\right)$. A coluna é equipada com $n$ tensiômetros nas profundidades $z_{1}, z_{2} \ldots z_{n}$. O solo na coluna, fechada no seu lado inferior, perde água por evaporação na sua superfície superior. Em $k$ diferentes instantes (tempos $t_{1}, t_{2}, \ldots, t_{k}$ ), são feitas observações dos potenciais matriciais nas $n$ posições munidas com tensiômetros, sendo o potencial matricial $h_{i, j}$ o valor obtido na profundidade $z_{i}$ e no tempo $t_{j}$. Analogamente a Schindler \& Müller (2006), supõe-se diminuição linear do conteúdo de água na coluna de baixo para cima. Com base na equação de Darcy-Buckingham, demonstra-se que, para essas condições, a condutividade hidráulica do solo $K\left(\mathrm{~m} \mathrm{~s}^{-1}\right)$ referente ao potencial matricial médio $h$ entre as profundidades $z_{i}$ e $z_{i+1}$ e no intervalo de tempo $t_{j}$ a $t_{j+1}$ é igual a:

$$
K(h)=\frac{V_{E}}{A \nabla \Delta t}\left[1-\frac{z_{i}+z_{i+1}}{2 L}\right]
$$

em que $\Delta t=t_{j+1}-t_{j}$ (s) é o intervalo de tempo de observação; $V_{E}\left(\mathrm{~m}^{3}\right)$ é o volume de água do solo evaporado durante o intervalo; e $\nabla$ é o gradiente hidráulico médio no intervalo, dado por:

$$
\nabla=\frac{\left(h_{i, j}-h_{i+1, j}\right)+\left(h_{i, j+1}-h_{i+1, j+1}\right)}{2 \Delta z}-1
$$

$\operatorname{com} \Delta z=z_{i+1}-z_{i}$.

\section{Retenção da água}

Nos $k$ instantes $\left(t_{1}, t_{2}, \ldots, t_{k}\right)$ em que se observam os potenciais matriciais, é realizada uma determinação da massa do conjunto, obtendo-se assim a variação da massa entre os tempos de observação. No fim do experimento, a massa da água restante no solo é determinada pelo método gravimétrico (secagem do solo a $105^{\circ} \mathrm{C}$ ). Essa massa final, somada às variações de massa entre as observações, permite calcular os $k$ massas de água $\left(m_{1}, m_{2}, \ldots, m_{k}\right)(\mathrm{kg})$ correspondentes a cada tempo de observação.

Para descrição matemática da curva de retenção, deve-se optar por uma equação funcional $\theta=\theta(h)$. No presente estudo, utilizou-se a equação descrita por van Genuchten (1980): 


$$
\Theta_{i, j}=\left[1+\left|\alpha h_{i, j}\right|^{n}\right]^{\frac{1}{n}-1}
$$

em que $\Theta_{i, j}=\left(\theta_{i, j}-\theta_{r}\right) /\left(\theta_{s}-\theta_{r}\right), \theta_{i, j}$ é o teor de água estimado pela equação na profundidade $z_{i}$ e no instante $t_{j}, \theta_{r}$ e $\theta_{s}$ são os teores de água residual e de saturação, respectivamente, e $\alpha\left(\mathrm{m}^{-1}\right)$ e $n$ são parâmetros empíricos da equação.

Por meio de um procedimento iterativo encontramse os valores dos parâmetros da equação que melhor coincidem com as observações experimentais. Inicialmente, atribuem-se valores arbitrários aos parâmetros, por exemplo, $\theta_{s}=0,5 \mathrm{~m}^{3} \mathrm{~m}^{-3}$, $\theta_{r}=0,1 \mathrm{~m}^{3} \mathrm{~m}^{-3}, \alpha=1,5 \mathrm{~m}^{-1} \mathrm{e} n=2,0$. Pela equação 3 , cada observação de potencial matricial é transformada num teor de água, e com os teores de água assim estimados para as $n$ posições com tensiômetros, a massa de água $\hat{m}_{j}(\mathrm{~kg})$ contida no solo no instante $j$ pode ser estimada pela expressão:

$$
\hat{m}_{j}=\rho_{a} \frac{A}{2}\left[\theta_{1, j}\left(z_{1}+z_{2}\right)+\sum_{i=2}^{n-1}\left[\theta_{i, j}\left(z_{i+1}-z_{i-1}\right)\right]+\theta_{n, j}\left(2 L-z_{n-1}-z_{n}\right)\right]
$$

em que $\rho_{a}\left(\mathrm{~kg} \mathrm{~m}^{-3}\right)$ é a densidade da água.

Os $n$ valores de $\theta_{i, j}$ no instante $t_{j}$ são então recalculados como:

$$
\theta_{i, j}{ }^{l}=\theta_{i, j}{ }^{l-1} \frac{m_{j}}{\hat{m}_{j}}
$$

em que $l$ representa o número da iteração. Com o novo conjunto de dados obtido $\left(h_{i, j}, \theta_{i, j}\right)$, os parâmetros da equação 3 são estimados novamente. O processo é repetido até a convergência, quando os parâmetros não se alteram mais entre duas iterações.

\section{EXPERIMENTO}

Uma amostra de terra da camada superficial de um Nitossolo Vermelho com densidade do solo, sob condições naturais de $1.200 \mathrm{~kg} \mathrm{~m}^{-3}$, foi coletada em uma área experimental da Escola Superior de Agricultura "Luiz de Queiroz" da Universidade de São Paulo (ESALQ/USP) em Piracicaba, São Paulo (22 ${ }^{\circ}$ $\left.42^{\prime} \mathrm{S}, 47^{\circ} 38^{\prime} \mathrm{W}\right)$. A amostra foi seca ao ar e peneirada em peneira com abertura de $2 \mathrm{~mm}$. Em seguida, transferiu-se o material peneirado para um anel de PVC, com $10 \mathrm{~cm}$ de altura, $14,5 \mathrm{~cm}$ de diâmetro interno e área superficial de $164 \mathrm{~cm}^{2}$. O material foi lentamente saturado com água a partir da base.

Após o processo de saturação com água, três tensiômetros de polímeros foram inseridos horizontalmente nas profundidades de $2,3\left(z_{1}\right), 4,9\left(z_{2}\right)$ e $7,6 \mathrm{~cm}\left(z_{3}\right)$, por meio de aberturas circulares previamente feitas no anel. Os tensiômetros de polímeros utilizados, fabricados pela Environmental Sciences Group, da Wageningen University, Holanda, foram similares aos usados por van der Ploeg et al. (2008) e De Rooij et al. (2009), com intervalo de medida entre 0 e - 160 m e precisão de medida do instrumento de 0,204 m. Esse tipo de tensiômetro é composto de um cone cerâmico maciço, um reservatório de aço onde o polímero fica alocado, um transdutor de pressão (Figura 1) e um armazenador de dados individual. Detalhes técnicos sobre o tensiômetro podem ser encontrados em Bakker et al. (2007).

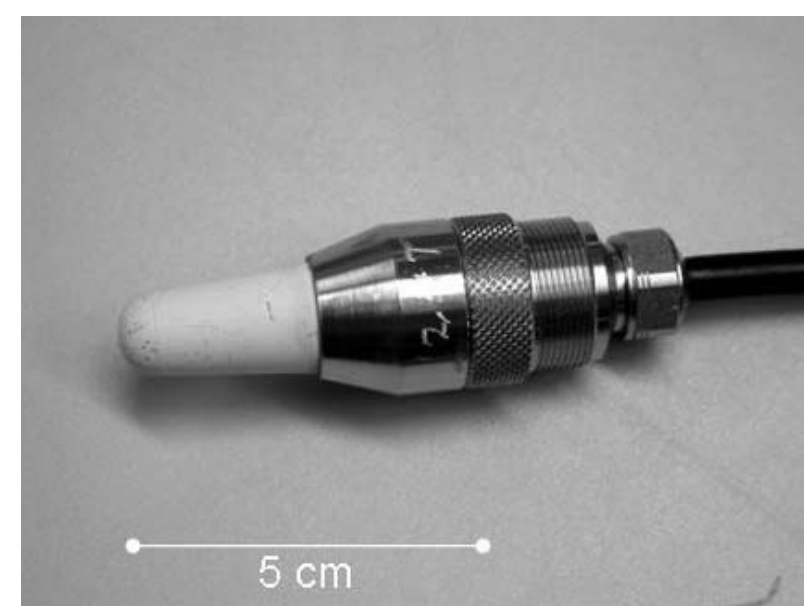

Figura 1. Ilustração de um tensiômetro de polímeros com dimensão aproximada, com a cápsula porosa (cerâmica clara) e o compartimento de aço contendo polímero e transdutor de pressão.

O anel com o material de solo com água munido com os tensiômetros foi colocado sobre uma balança de precisão (Tecnal Mark K16, com capacidade 16 kg e resolução de $0,1 \mathrm{~g}$ ), vedando-se a superfície inferior, para que não houvesse vazamento de água e a evaporação ocorresse somente pela superfície superior do anel. O laboratório foi mantido em temperatura e umidade relativa ambientes. O potencial matricial nas três profundidades $\left(h_{i, j}\right)$ e a massa do conjunto $m_{j}$ foram medidos automaticamente a cada $30 \mathrm{~min}$. As medidas foram finalizadas quando o tensiômetro superior atingiu o valor de potencial matricial de aproximadamente $-150 \mathrm{~m}$.

A cada intervalo de observação, $V_{E}$ (equação 1) foi calculado a partir da variação de massa $\Delta m=m_{l}-m_{j-1}(\mathrm{~kg})$ da amostra e a densidade da água $\rho_{a}\left(\mathrm{~kg} \mathrm{~m}^{-3}\right)$ :

$$
V_{E}=-\frac{\Delta m}{\rho_{a}}
$$

A condutividade hidráulica para cada intervalo de observação foi calculada pela equação 1 . Os parâmetros da equação da curva de retenção (equação 3) foram obtidos conforme descrito.

O procedimento completo foi executado por três vezes (experimentos I, II e III). 


\section{RESULTADOS E DISCUSSÃO}

A inspeção dos dados obtidos de potencial matricial mostrou que, na fase inicial do experimento (primeiros dias), o movimento de água na amostra não se apresentava unidirecional, isto é, enquanto na parte superior do cilindro a água ascendia por causa da evaporação, na parte inferior o movimento era descendente, devido à ação gravitacional. Embora o material tenha sido saturado com água inicialmente, durante a instalação dos tensiômetros houve pequena perda de água, o que levou à redistribuição da água na amostra. Esse fato fez com que o cálculo confiável do $K(h)$ pela equação 1 para esses dados não fosse possível. Por essa razão, optou-se por utilizar os dados somente a partir do momento em que o movimento se tornou unidirecional (de baixo para cima) no cilindro inteiro, verificado pelos valores dos potenciais totais nas posições dos tensiômetros.

O início do movimento unidirecional foi observado 2 a 3 dias após o início do processo de evaporação, quando os potenciais matriciais eram da ordem de -1 a -3 m. Assim, pode-se dizer que o método utilizado não é adequado para determinação da condutividade hidráulica na faixa muito úmida $(h>-1 \mathrm{~m})$. Outros fatores experimentais vêm ao encontro dessa constatação: a precisão de medida dos tensiômetros de polímeros em torno de $0,2 \mathrm{~m}$ faz com que observações na faixa muito úmida contenham erro relativo muito grande; e a utilização de material de solo peneirado e deformado não garante a preservação da macroestrutura do solo, sendo o método não adequado para determinar propriedades hidráulicas do solo muito úmido.

As densidades do solo nos experimentos I, II e III foram de $1.163,1.193 \mathrm{e} 1.105 \mathrm{~kg} \mathrm{~m}^{-3}$, respectivamente. As curvas de retenção nos três experimentos são representadas na figura 2 , juntamente com os respectivos parâmetros de ajuste da equação de van Genuchten (1980). Foram necessárias menos de 10 iterações de cálculo para que os parâmetros da equação 3 convergissem ao valor final.

Os valores de $\theta_{s}$ e os de $\alpha$ foram bastante diferentes entre os três experimentos. Os valores de $\theta_{s}$ foram baixos quando confrontados com a densidade do solo, especialmente nos experimentos II e III. $\mathrm{Na}$ interpretação desses fatos, deve-se levar em consideração que o ajuste da equação 3 foi feito sem a inclusão de valores próximo à saturação, apenas para $h$ menor que $-1 \mathrm{~m}$, aproximadamente. Assim, a validade da equação ajustada corresponde somente à faixa de valores observados, e $\theta_{s}$, da mesma forma que $\alpha, n$ e $\theta_{r}$, é um mero parâmetro de ajuste da equação, sem significado físico.

Os resultados para a relação entre a condutividade hidráulica $K$ e o potencial matricial $h$ estão representados na figura 3. O decréscimo de $K \operatorname{com} h$ foi semelhante nos três experimentos, exceto na camada de solo entre as profundidades $z_{2}$ e $z_{3}$ da terceira repetição. Nessa camada, os valores de $K$ foram mais altos em comparação com a camada superior de solo durante todo o experimento, possivelmente devido a uma compactação diferente do solo.

Os valores de $K(\theta)$ podem apresentar relação exponencial com o teor de água. Nesses casos, a função $K(\theta)$ pode convenientemente ser expressa pela equação:

$$
K(\theta)=K^{*} \cdot e^{\gamma \theta}
$$
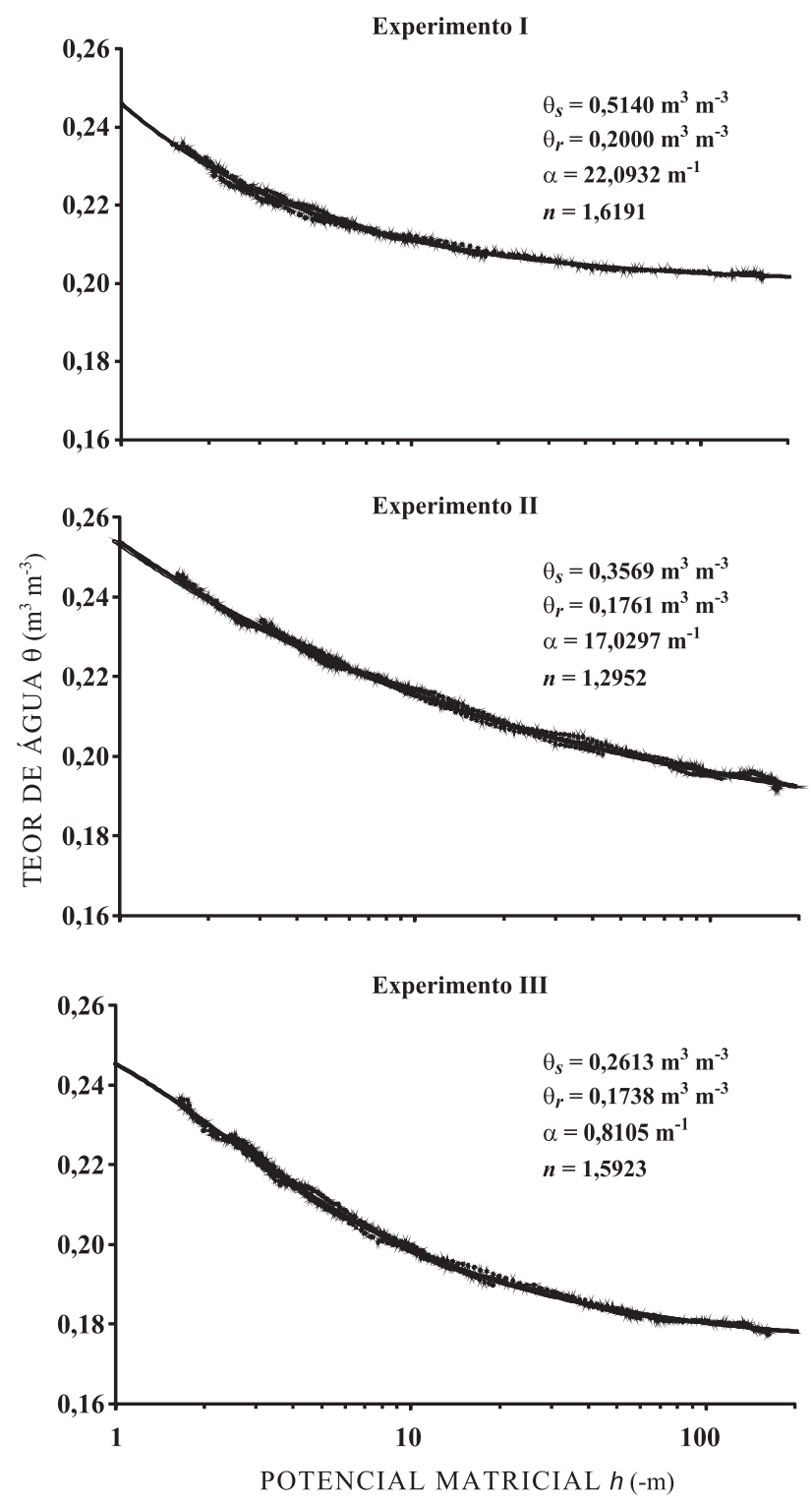

Figura 2. Teor de água em função do potencial matricial (curvas de retenção) para os experimentos I, II e III. Os pontos representam os valores obtidos experimentalmente; a linha é a equação 3 ajustada. 


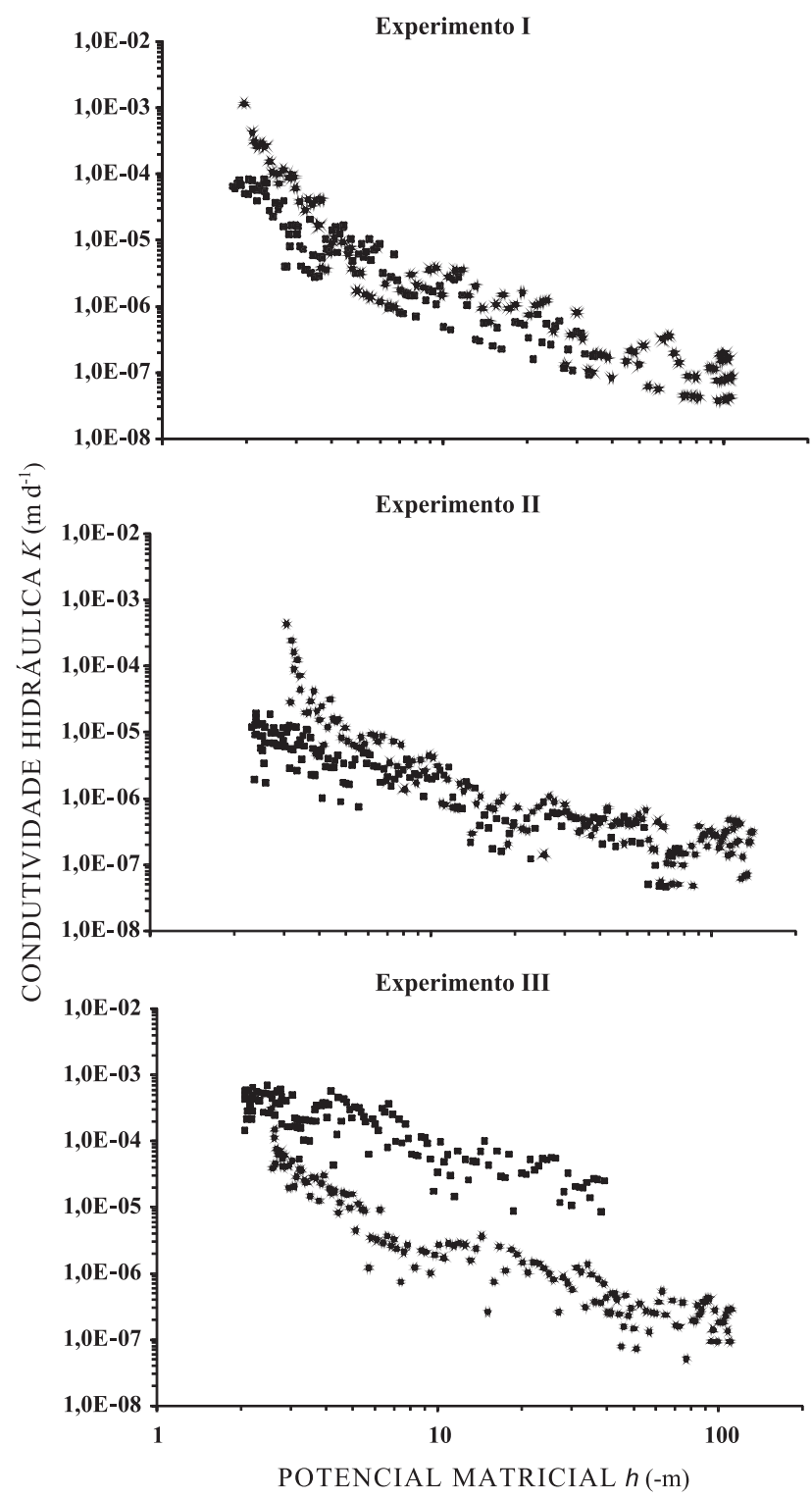

Figura 3. Condutividade hidráulica em função do potencial matricial para os experimentos I, II e III. Círculos representam dados obtidos entre as profundidades $z_{1}-z_{2}$; quadrados são dados obtidos entre as profundidades $z_{2}-z_{3}$.

em que $\gamma$ é uma constante adimensional e $K^{*}\left(\mathrm{~m} \mathrm{~d}^{-1}\right)$ é o valor hipotético da condutividade hidráulica quando o teor de água equivale a zero. A relação entre a condutividade hidráulica $K$ e o teor de água no solo estimado pela equação 3 em função dos potenciais matriciais observados é apresentada na figura 4. Os valores de $\gamma$ são da ordem de 100 a 250 - mesma ordem de grandeza observada num experimento de campo no mesmo solo por De Jong Van Lier \& Libardi (1999).
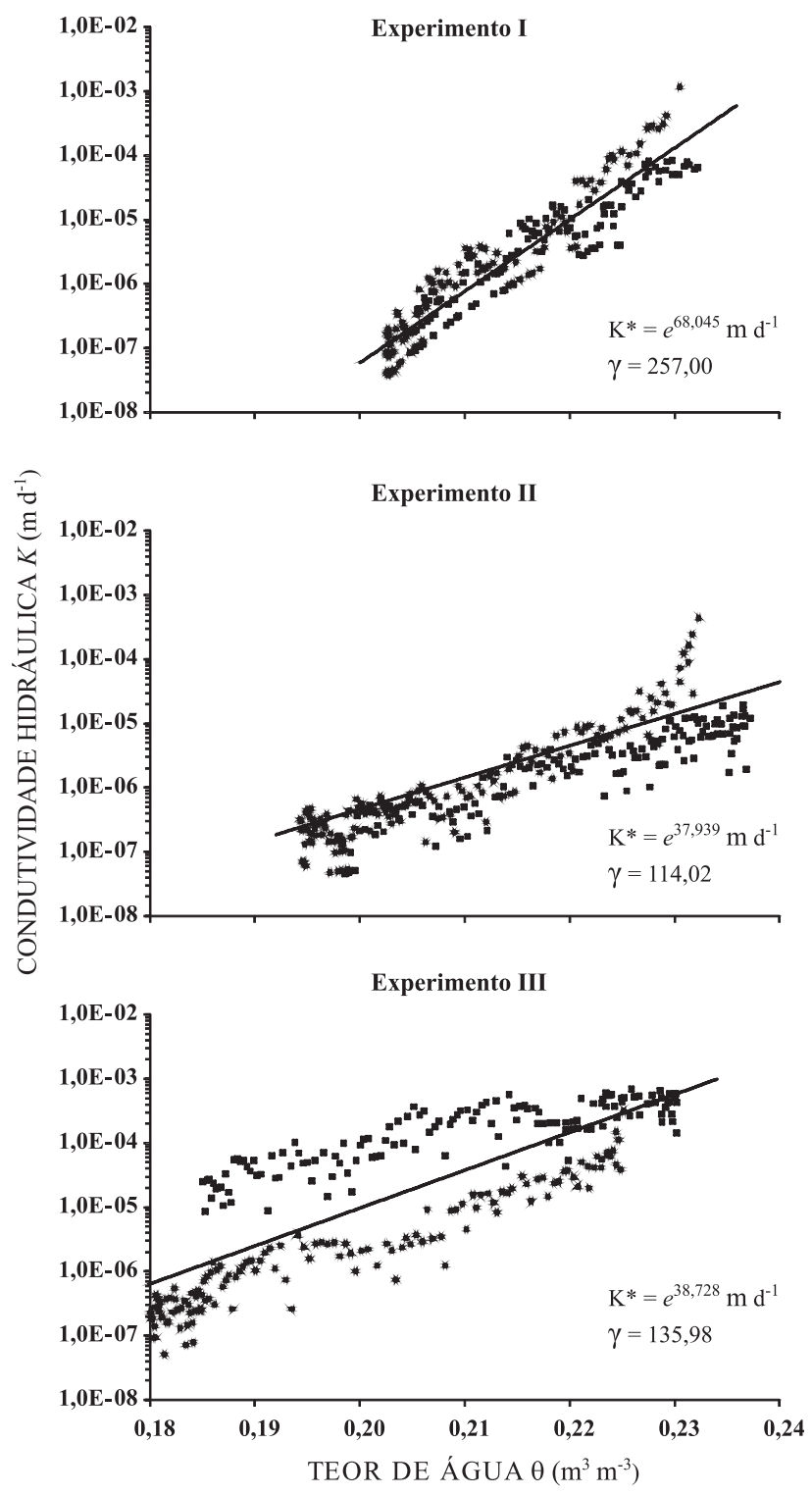

Figura 4. Condutividade hidráulica em função do teor de água para os experimentos I, II e III. Círculos representam dados obtidos entre as profundidades $z_{1}-z_{2}$; quadrados são dados obtidos entre as profundidades $z_{2}-z_{3}$; e a linha é a equação 7 ajustada.

\section{CONCLUSÕES}

1. O tensiômetro de polímeros, um instrumento novo, permite medir diretamente potenciais matriciais da água no solo até valores próximos ao do ponto de murcha permanente, $-150 \mathrm{~m}$.

2. A utilização de tensiômetros de polímeros em experimentos de evaporação com colunas de solo permite a determinação das relações entre 
condutividade hidráulica, teor de água e potencial matricial na faixa de potenciais matriciais entre aproximadamente $-1 \mathrm{e}-150 \mathrm{~m}$.

3. Observações no solo mais úmido (potenciais matriciais menos negativos que $-1 \mathrm{~m}$ ) não puderam ser feitas com confiança, pelo fato de os perfis de potencial nos cilindros não indicarem movimento unidirecional nos primeiros dias do experimento.

\section{AGRADECIMENTO}

A Hermanus P. A. Gooren, da Universidade de Wageningen, Holanda, pelo treinamento no uso dos tensiômetros de polímeros.

\section{LITERATURA CITADA}

ARYA, L.M. Wind and hot-air methods. In: DANE, J.H. \& TOPP, D G.C., eds. Methods of soil analysis: Physical methods. Madison, Soil Science Society of America, 2002. Part 4. p.916-920.

BAKKER, G.; van DER PLOEG, M.J.; DE ROOIJ, G.H.; HOOGENDAM, C.W.; GOOREN, H.P.A.; HUISKES, C.; KOOPAL, L.K. \& KRUIDHOF, H. New polymer tensiometers: Measuring matric pressures down to the wilting point. Vadose Zone J., 6:196-202, 2007.

DANE, J.H. \& TOPP, G.C., eds. Methods of soil analysis: Physical methods. Madison, Soil Science Society of America, 2002. Part 4. 1692p. (SSSA Book Series, 5)

DE JONG VAN LIER, Q. \& LIBARDI, P.L. Variabilidade dos parâmetros da equação que relaciona a condutividade hidráulica com a umidade do solo no método do perfil instantâneo. R. Bras. Ci. Solo, 23:1005-1014, 1999.

DE ROOIJ, G.H.; VAN DER PLOEG, M.J.; GOOREN, H.P.A.; BAKKER, G.; HOOGENDAM, C.W.; HUISKENS, C.; KRUIDHOF, H. \& KOOPAL, L.K. Measuring very negative water potentials with polymer tensiometers: principles, performance and applications. Biologia, 64:438 442, 2009 .
PETERS, A. \& DURNER, W. Simplified evaporation method for determining soil hydraulic properties. J. Hydrol., $356: 147-162,2008$

SCHINDLER, U. Ein schnellverfahren zur messung der wasserleitfähigkeit im teilgesättigten boden an stechzylinderproben. Arch. Acker-u. Pflanz. Bodenkd., 24:1-7, 1980.

SCHINDLER, U.; BOHNE, K. \& SAUERBREY, R. Comparison of different measuring and calculating methods to quantify the hydraulic conductivity of unsaturated soil. Z. Pflanzenernähr. Bodenkd., 148:607-617, 1985.

SCHINDLER, U. \& MÜLLER, L. Simplifying the evaporation method for quantifying soil hydraulic properties. J. Plant Nutr. Soil Sci., 169:623-629, 2006.

SCHNEIDER, K.; IPPISCH, O. \& ROTH, D.K. Novel evaporation experiment to determine soil hydraulic properties. Hydrol. Earth Syst. Sci., 10:817-827, 2006.

ŠIMÙNEK, J.; WENDROTH, O. \& van GENUCHTEN, M.T. Parameter estimation analysis of the evaporation method for determining soil hydraulic properties. Soil Sci. Soc. Am. J., 62:894-905, 1998.

van DER PLOEG, M.J.; GOOREN, H.P.A.; BAKKER, G. \& DE ROOIJ, G.H. Matric potentials measurements by polymer tensiometers in cropped lysimeters under water-stressed conditions. Vadose Zone J., 7:1048-1054, 2008.

van GENUCHTEN, M.T. A closed-form equation for predicting the hydraulic conductivity of unsaturated soils. Soil Sci. Soc. Am. J., 44:892-897, 1980.

WENDROTH, O.; EHLERS, W.; HOPMANS, J.W.; KLAGE, H.; HALBERTSMA, J. \& WÖSTEN, J.H.M. Reevaluation of the evaporation method for determining hydraulic functions in unsaturated soils. Soil Sci. Soc. Am. J., 57:1436-1443, 1993.

WIND, G.P. Capillary conductivity data estimated by a simple method. In: RIJTEMA, P.E. \& WASSINK, H., eds. Water in the unsaturated zone. v.1; Proceedings of the Wageningen Symposium, 19-23 June 1968. Int. Assoc. Sci. Hydrol. Publ. (IASH), Gentbrugge, The Netherlands and UNESCO, Paris, 1968. 